\title{
Proposal and verification of a new visible light emitter based on wide band gap II-VI semiconductors
}

\author{
M. C. Phillips, M. W. Wang, J. F. Swenberg, J. O. McCaldin, and T. C. McGill \\ T. J. Watson, Sr...Laboratory of Applied Physics, 128-95 California Institute of Technology, \\ Pasadena, California 91125
}

(Received 8 June 1992; accepted for publication 28 August 1992)

\begin{abstract}
We propose a new device structure for obtaining visible light emission from wide band gap semiconductors. This heterojunction structure avoids ohmic contacting problems by using only the doping types which tend to occur naturally in II-VI semiconductors, while using a novel injection scheme to obtain efficient minority carrier injection into the wider band gap semiconductor. To verify this proposal we have fabricated green light emitting structures using $n$-CdSe and $p$-ZnTe regions separated by a graded $\mathrm{Mg}_{x} \mathrm{Cd}_{1-x} \mathrm{Se}$ injection region. Room temperature electroluminescence spectra from these devices demonstrate the effectiveness of the injection scheme, while the current-voltage characteristics show the merits of avoiding difficult ohmic contacts. We further show how the structure can be extended to blue wavelengths and beyond by opening up the band gap of the $\mathrm{ZnTe}$ recombination region with a $\mathrm{Mg}_{y} \mathrm{Zn}_{1-y} \mathrm{Te}$ alloy.
\end{abstract}

In the last year we have seen a flurry of activity in the development of visible light emitters fabricated from wide hand gap II-VI semiconductors. ${ }^{1-3}$ Based on work by a number of groups on nonequilibrium, $p$-type doping of ZnSe with $\mathbf{N},{ }^{4-6}$ Haase et al. ${ }^{1}$ successfully fabricated the first blue laser diodes. Other authors have followed up on this pioneering effort and have demonstrated a number of different aspects of these devices, ${ }^{2,3}$ but little progress has been reported on the major problem of obtaining stable layers of $p-\mathrm{ZnSe}$ and $p$-ZnSSe with net doping above 2 $\times 10^{18} / \mathrm{cm}^{3}$. The combination of this doping level with a Schottky barrier of over $1 \mathrm{~V}$ has made it impossible to form satisfactory ohmic contacts. This perhaps fundamental difficulty has led to operating voltages around $15 \mathrm{~V}$ for light emitting diodes (LEDs) and lasers. Problems dissipating the resulting high power inputs have prevented continuous-wave operation at room temperature and have resulted in short lifetimes even for lasers operating at $77 \mathrm{~K}$. If these doping problems cannot be solved, they may preclude the development of commercially viable devices based on nitrogen-doped $\mathrm{ZnSe}$ and $\mathrm{ZnSSe}$.

In this letter we propose and demonstrate an entirely new device structure for short wavelength LEDs and lasers. The essence of the device is a $p-n$ heterojunction separated by a thin region which includes both smooth grading and an abrupt heterojunction. This "graded injector" allows injection of minority carriers into the wider band gap material, while injection into the narrower band gap material remains blocked by a band offset. By choosing a $p-n$ heterojunction that uses only the doping types which tend to occur naturally, we avoid problems with ohmic contacts.

Tellurides are the only wide band gap II-VI's which are easily doped $p$ type, and $\mathrm{CdSe} / \mathrm{ZnTe}$ is the only closely lattice matched $p-n$ heterojunction among II-VI binaries. Though CdSe occurs naturally in wurtzite form, it grows readily in $\mathrm{Zn}$-blende form on (100) $\mathrm{ZnTe}$, with a lattice constant $0.44 \%$ smaller than that of $\mathrm{ZnTe}{ }^{7}$ Furthermore, CdSe can be doped $n$ type to carrier concentrations of at least several $\times 10^{19} / \mathrm{cm}^{3}$ with Al. A simple $n$-CdSe $/ p-\mathrm{ZnTe}$ heterojunction, however, is a very poor light emitter due to the unfavorable type II band alignment-the valence band of CdSe lies $0.64 \mathrm{eV}$ below that of $\mathrm{ZnTe}$, and the conduction band lies $1.15 \mathrm{eV}$ below that of $\mathrm{ZnTe} .^{8}$ In forward bias, large accumulations of electrons and holes form at the interface, thus driving nonradiative recombination. Simple grading from CdSe to $\mathrm{ZnTe}$ could reduce the accumulation regions, but most of the current would be holes injected into the CdSe due to the larger barrier to injection of electrons into the ZnTe.

To allow injection of electrons into the $\mathrm{ZnTe}$ while blocking the injection of holes into the CdSe, we need smooth grading in the conduction band and an abrupt discontinuity in the valence band. The flatband diagram in the upper half of Fig. 1 shows how this can be accomplished by adding $\mathrm{Mg}$ to the CdSe to raise the conduction band of the CdSe up to that of $\mathrm{ZnTe}$. The abrupt change in anion from $\mathrm{Se}$ to $\mathrm{Te}$ at the interface gives an abrupt discontinuity in the valence band which blocks holes. The lattice constant of pure $\mathrm{Zn}$-blende $\mathrm{MgSe}$ is $5.89 \AA^{9}$-about $3.6 \%$ less than $\mathrm{ZnTe}$-so alloying with $\mathrm{Mg}$ introduces strain. The various estimates and extrapolations of the band gap of pure $\mathrm{MgSe}$ range from 3.6 to $5.6 \mathrm{eV}, 9,10$ but we believe a good estimate for the $\mathrm{Zn}$-blende form is about $4.1 \mathrm{eV}$. To grade the conduction band of the $\mathrm{Mg}_{x} \mathrm{Cd}_{1-x} \mathrm{Se}$ up to the conduction band of the $\mathrm{ZnTe}$ requires a maximum $\mathrm{Mg}$ concentration of about $x=0.52$, assuming the common anion rule applies as suggested in Fig. 1. Thus at the peak of the grading we have a lattice mismatch of $1.9 \%$ and the graded region must be kept thin to stay under the critical thickness. Detailed simulations of the device show that the grading can occur over very short distances.

Figure 1(b) shows the result of a simulation of the device structure shown in Fig. 1(a). ${ }^{11}$ The device turns on like a normal diode as the applied bias approaches the band gap of the $\mathrm{ZnTe}$. The band diagram is shown with the device biased at $2.0 \mathrm{~V}$. At this bias the remaining barrier to injection of electrons is about $0.3 \mathrm{~V}$, so there is substantial injection into the $\mathrm{ZnTe}$. The accumulations of electrons and holes are much smaller than for the bare $n-\mathrm{CdSe} / p$ - 

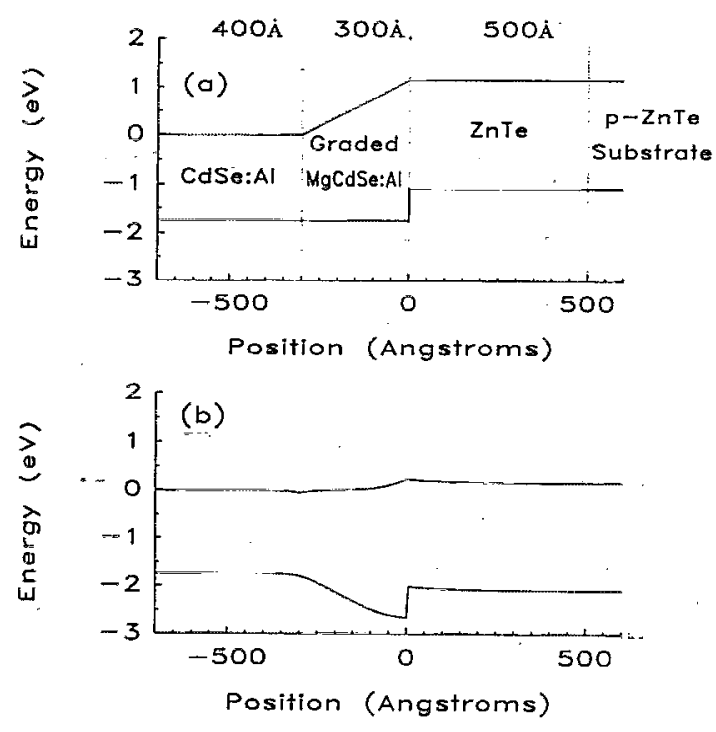

FIG. 1. (a) Flatband diagram of a simple graded injection device. (b) Calculated band diagram for same device biased at $2.0 \mathrm{~V}$.

$\mathrm{ZnTe}$ heterojunction, and the accumulations are spatially separated by approximately the width of the grading, thus inhibiting nonradiative recombination.

To demonstrate this graded injector, we grew the green LED structure shown in Fig. 1(a) using procedures similar to those we have reported elsewhere for growing $n$ $\mathrm{CdSe} / p-\mathrm{ZnTe}$ heterojunctions by molecular beam epitaxy (MBE). ${ }^{12}$ For the grading we added an effusion cell loaded with elemental $\mathrm{Mg}$. We calibrated the peak concentration of the $\mathrm{Mg}$ by x-ray photoemission spectroscopy on bulk layers of $\mathrm{Mg}_{x} \mathrm{Cd}_{1-x} \mathrm{Se}$ and checked the accuracy of these measurements with electron microprobe analysis. Since there are some uncertainties in the dependence of the band gap of the $\mathrm{Mg}_{x} \mathrm{Cd}_{1-x} \mathrm{Se}$ on $x$, and since extending the grading slightly too high is not a problem, we aimed for a maximum $\mathrm{Mg}$ concentration of $x=0.6$. This overshoot is also more than enough to allow for the decrease in the band gap of the $\mathrm{Mg}_{x} \mathrm{Cd}_{1-x} \mathrm{Se}$ alloy due to strain. To obtain the grading we simply shut off the power to the $\mathrm{Mg}$ effusion cell when the $\mathrm{Mg}, \mathrm{Cd}$, and Se shutters opened. We had previously measured the decrease in the Mg flux after shutting off the power to the cell using an ion tube flux monitor and a residual gas analyzer. We then adjusted the CdSe growth rate so that the grading would occur over $200-300$ $\AA$. Device simulations show that the exact shape of the grading is not important. In situ reflection high energy electron diffraction showed no evidence of a change from $\mathrm{Zn}$-blende structure during the growth of the $\mathrm{Mg}_{x} \mathrm{Cd}_{1-x} \mathrm{Se}$ or CdSe. Further structural characterization is in progress.

Figure 2 shows a comparison of the room temperature electrical characteristics of our graded- $\mathrm{Mg}_{x} \mathrm{Cd}_{1-x} \mathrm{Se}$ device with the $\mathrm{ZnSe}: \mathrm{N}$ blue-green laser of Haase et al. ${ }^{1}$ and the ZnSe:N LED with an inverted structure reported by Jeon et $a .^{13}$ At less than $10 \mathrm{~V}$, the current densities in the graded device are hundreds of $\mathrm{A} / \mathrm{cm}^{2}$. This data was taken at room temperature without pulsing and without special heat sinking. While these current-voltage characteristics compare quite favorably with the $\mathrm{ZnSe}: \mathrm{N}$ devices, the op-

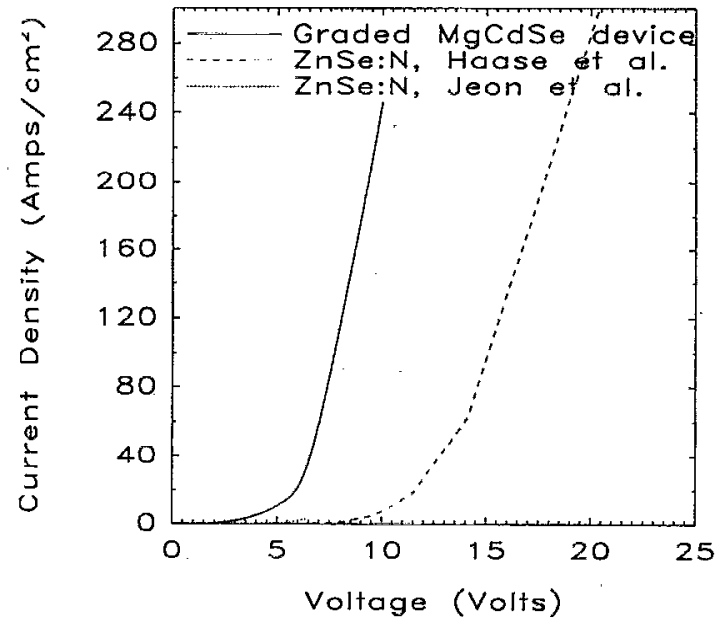

FIG. 2. Room temperature electrical characteristics of the graded$\mathrm{Mg}_{x} \mathrm{Cd}_{1-x}$ Se device described in Fig. 1 compared to $\mathrm{ZnSe:N}$ laser diode (pulsed) (Ref. 1) and a ZnSe:N LED (Ref. 13). $\mathrm{Mg}_{x} \mathrm{Cd}_{1-x}$ Se device is a mesa $110 \mu \mathrm{m}$ in diameter.

erating voltage is still much higher than predicted by our simulations. This is due to several shortcomings of this primitive stage of our device. Oriented, single crystal $\mathrm{ZnTe}$ substrates are not yet a standard commercial item, and the best substrates we have obtained have poor structural quality and a resistivity of about $2 \Omega \mathrm{cm}$. This produces the large series resistance evident in Fig. 2. Second, this device was grown without an effective $p$-type dopant for $\mathrm{ZnTe}$, so we had to rely on the $p$ typeness of the substrate and grow only a thin buffer layer. The slower-than-expected turn-on of our device is probably due to charging of traps in the undoped buffer and possibly also in the $\mathrm{Mg}_{x} \mathrm{Cd}_{1-x} \mathrm{Se}$, which we have still not adequately characterized. In spite of the low carrier concentration of the $\mathrm{ZnTe}$ substrate (about $3 \times 10^{16} / \mathrm{cm}^{3}$ ), satisfactory ohmic contacts can be made by flash-evaporating $\mathrm{Sb}_{2} \mathrm{Te}_{3}$, sometimes followed by an anneal at $230^{\circ} \mathrm{C}$. It seems likely that this produces high doping in a very thin layer at the contacting surface.

The electroluminescence spectrum in Fig. 3 shows that we are obtaining electron injection into the $\mathrm{ZnTe}$. The large peak is band-edge emission from $\mathrm{ZnTe}$. Since this device has such a thin buffer layer, most of the recombination takes place in the substrate, which is of poor structural quality and contains an isoelectronic oxygen recombination center. The weaker signal in the red is from this recombination center in the substrate. There is never any luminescence from the $\mathrm{CdSe}$, indicating that the graded injector is blocking holes as desired.

By extending the grading to higher $\mathrm{Mg}$ concentrations, electrons can be injected into an alloy with a band gap larger than that of $\mathrm{ZnTe}$. Figure 4 shows how this could be done by expanding the band gap of the $\mathrm{ZnTe}$ recombination region by alloying with $\mathrm{Mg} . \mathrm{Mg}_{y} \mathrm{Zn}_{1-y} \mathrm{Te}$ alloys have been studied more extensively than $\mathrm{Mg}_{x} \mathrm{Cd}_{1-x} \mathrm{Se}$ alloys, and the band gap of pure $\mathrm{MgTe}$ is about $3.47 \mathrm{eV} .{ }^{14}$ Thus the band gaps of $\mathrm{Mg}_{x} \mathrm{Cd}_{1-x} \mathrm{Se}$ and $\mathrm{Mg}_{y} \mathrm{Zn}_{1-y} \mathrm{Te}$ reach easily to the violet; the limit of the wavelengths possible will be determined by how much lattice mismatch can be tol- 


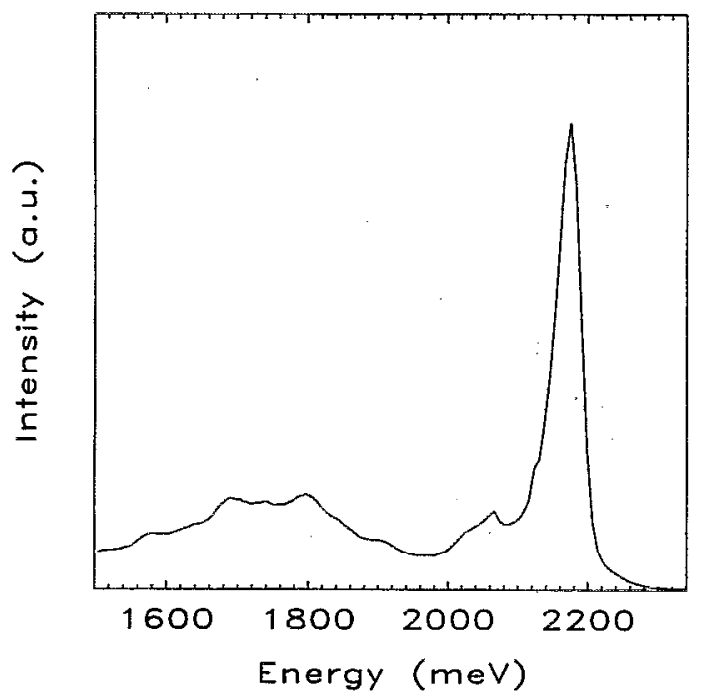

FIG. 3. Room temperature electroluminescence spectrum of the graded$\mathrm{Mg}_{x} \mathrm{Cd}_{1-x}$ Se LED described in Fig. 1. Broad peak centered around 1.8 $\mathrm{eV}$ is from an impurity center present in the substrate.

erated. Furthermore, this graded injected scheme is not limited to this material system and could be used to overc ome unfavorable band alignments in other $p$ - $n$ heterojunction systems.

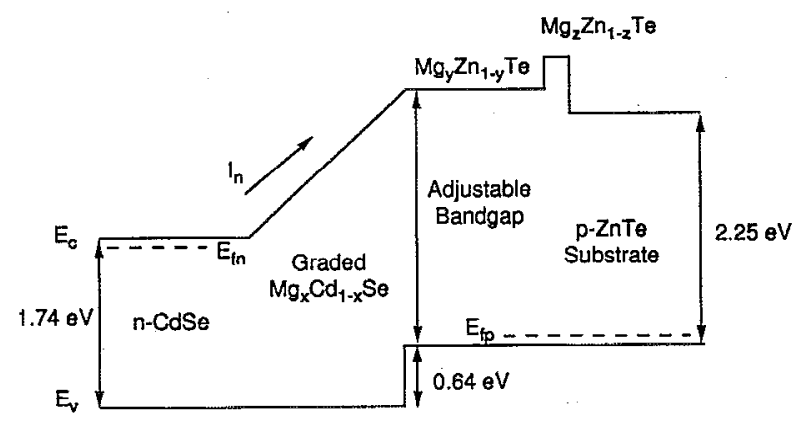

FIG. 4. Flatband diagram of blue LED made by extending grading to inject into a $\mathrm{Mg}_{y} \mathrm{Zn}_{1-y} \mathrm{Te}$ recombination region.
Work currently in progress involves implementation of designs for both LEDs and lasers. We are presently characterizing a $p$-type dopant for MBE growth of $\mathrm{ZnTe}$, which should overcome several of the major flaws in the primitive device described in this letter. While it is surely true that various other materials difficulties require solution, this seems to be a very promising approach to making short wavelength LEDs and lasers.

The authors would like to acknowledge valuable discussions with D. H. Chow and E. T. Yu. The Defense Advanced Research Projects Agency provided critical support during the conception and development of this device concept with Contract Nos. N00014-90-J-1742 and N00014-86-K-0841. We have also benefited from the patient and helpful monitoring of this program by the Office of Naval Research through L. R. Cooper.

${ }^{1}$ M. A. Haase, J. Qiu, J. M. Depuydt, and H. Cheng, Appl. Phys. Lett. 59, 1272 (1991).

${ }^{2}$ H. Jeon, J. Ding, W. Patterson, A. V. Nurmikko, W. Xie, D. C. Grillo, M. Kobayashi, and R. L. Gunshor, Appl. Phys. Lett. 59, 3619 (1991). ${ }^{3}$ H. Jeon, J. Ding, A. V. Nurmikko, W. Xie, D. C. Grillo, M. Kobayashi, R. L. Gunshor, G. C. Hua, and N. Otsuka, Appl. Phys. Lett. 60 , 2045 (1992).

${ }^{4}$ K. Ohkawa, T. Mitsuyu, and O. Yamazaki, J. Cryst. Growth 86, 329 (1988).

${ }^{5}$ R. M. Park, M. B. Troffer, C. M. Rouleau, J. M. DePuydt, and M. A. Haase, Appl. Phys. Lett. 57, 2127 (1990).

${ }^{6}$ K. Ohkawa, T. Karasawa, and T. Mitsuyu, J. Cryst. Growth 111, 797 (1991).

${ }^{7}$ N. Samarth, H. Luo, J. K. Furdyna, S. B. Qadri, Y. R. Lee, A. K. Ramdas, and N. Otsuka, Appl. Phys. Lett. 54, 2680 (1989).

${ }^{8}$ E. T. Yu, M. C. Phillips, J. O. McCaldin, and T. C. McGill, J. Vac. Sci. Technol. B 9, 2233 (1991).

${ }^{9}$ H. Okuyama, K. Nakano, T. Miyajima, and K. Akimoto, Jpn. J. Appl. Phys. 30, L1620 (1991).

${ }^{10}$ W. H. Strehlow and E. L. Cook, J. Phys. Chem. Ref. Data 2, 163 (1973).

${ }^{11}$ M. W. Wang, M. C. Phillips, J. F. Swenberg, E. T. Yu, J. O. McCaldin, and T. C. McGill (unpublished).

${ }^{12}$ M. C. Phillips, E. T. Yu, Y. Rajakarunanayake, J. O. McCaldin, D. A. Collins, and T. C. McGill, J. Cryst. Growth 111, 820 (1991).

${ }^{13}$ H. Jeon, J. Ding, A. V. Nurmikko, W. Xie, M. Kobayashi, and R. L. Gunshor, Appl. Phys. Lett. 60, 892 (1992).

${ }^{14}$ S. G. Parker, A. R. Reinberg, J. E. Pinnell, and W. C. Holton, J. Electrochem. Soc. 118, 979 (1971). 